\title{
Vasopressin test in cases of optic atrophy and optic neuritis
}

\author{
P. O. LUNDBERG，P. O. OSTERMAN, AND L. WIDE \\ From the Department of Neurology and Department of Clinical Chemistry, University Hospital, \\ Uppsala, Sweden
}

\begin{abstract}
SUMMARY The vasopressin test gave pathological results in 12 cases of optic atrophy and normal results in three cases. One of the patients with a pathological response had Leber's disease and three had tobacco-alcoholic amblyopia, while in the rest the optic atrophy was of uncertain origin. In the cases with normal results the aetiology was also unclear. The Metopirone test was normal in 13 cases and pathological in only one case of optic atrophy. In three out of five patients with optic neuritis the vasopressin test gave pathological responses. The high frequency of pathological vasopressin tests in patients with optic lesions indicates a simultaneous disturbance of the hypothalamo-hypophyseal function. The background to this might be a disturbed vascular supply. The vasopressin test was of no help in diagnosing tumours as a cause of optic atrophy.
\end{abstract}

In impairment of vision caused by processes affecting one or both of the optic nerves or the optic chiasm, tumours comprise an important problem of diagnosis. Hypophyseal tumours, adenomas, and craniopharyngeomas can be revealed in most cases by radiographs of the skull, while other forms of tumour-for example, suprasellar meningiomascan be difficult to diagnose even with the aid of encephalography and carotid angiography. Sometimes surgical exploration is therefore unavoidable in spite of negative radiographic findings. Since the prognosis with regard to regression of visual impairment and visual field defects is highly dependent upon an early diagnosis of the tumour (see, for example, Bohm and Dolk, 1969), it is desirable to find additional aids to diagnosis.

Signs of endocrine dysfunction caused by pressure on the hypothalamus and/or hypophysis are common and early findings in tumours of the sellar region. Even purely suprasellar processes can give signs of endocrine insufficiency at an early stage. Suprasellar meningioma has thus been found to give a pathological result in the Metopirone test without the tumour having direct influence on the hypophysis (Lundberg and Hugosson, 1966).

In order to determine the value of the vasopressin and Metopirone test for differentiating optic atrophy caused by a tumour in the sellar region from optic atrophy of another origin (hereditary, toxic, or infectious), these tests were conducted (1) on a group of patients with optic atrophy of different origins, where tumours had been excluded, and (2) on a group of patients with optic neuritis. A number of patients with visual impairment either caused by diseases in the eye or of purely mental origin (hysterical amblyopia) served as controls for the vasopressin test.

PATIENTS

CONTROL GROUP FOR VASOPRESSIN TEST The patients in this group were treated in the same ward and in exactly the same way as those in the study groups. A careful examination was made in order to exclude signs of organic nervous disease or endocrine disorder. None of the patients were given any medical treatment at the time of the tests. The patients were selected so as to comprise cases of visual impairment not caused by diseases of the visual pathways (optic nerve, chiasm, or optic tract). The control group consisted of two cases of myopia, one of cataract, one of degeneration of the macula, one of benign pseudopapillitis, and two of hysterical amblyopia.

PATIENTS Group I: 15 patients with optic atrophy One of these patients had Leber's disease with an acute onset of bilateral visual impairment, bilateral central scotoma, and history of heredity; there was also some abuse of alcohol in this case.

Three of the patients had so called tobacco-alcoholic amblyopia. These patients also had bilateral optic atrophy and central scotoma, but the visual impairment occurred subacutely or insidiously.

In 11 patients there was optic atrophy of uncertain origin. Three of these had bilateral symptoms. In six 
cases the visual impairment had an acute onset or occurred in attacks, while in five cases there was a more insidious course. The most common visual field defects were enlarged blind spot (three cases), central scotoma (three cases), and visual field reduction, mainly inferonasally (two cases - one bilateral). One patient had a pronounced concentric visual field defect, and in two patients the visual field was essentially normal.

The duration of the disease before the time of the test varied between one month and 35 years. Tumours as a cause of optic atrophy were excluded with the aid of the course of the disease, radiography of the skull and encephalography (all cases), carotid angiography (10 patients), and, in three cases, by neurosurgical exploration.

GROUP II: Five patients with optic neuritis In one case the symptoms were bilateral. The diagnosis of multiple sclerosis was considered as certain in all cases, and was made from the course of the neuritis, the occurrence of periodic attacks with other neurological symptoms, and examination of the cerebrospinal fluid. None of these patients had been treated previously with ACTH or cortisone.

In none of the patients in group I or group II were there any other signs of involvement of the central or peripheral nervous system, and neither were there any signs of diabetes. At the time of the tests all patients were being treated at the Department of Neurology, University Hospital, Uppsala, and none of them was having any other medicines than vitamins.

METHODS The vasopressin test was performed in all cases; the method used has been described previously (Lundberg and Wide, 1969). Ten international units of synthetic lysine-8-vasopressin (Sandoz) was infused in one hour, between 9 a.m. and 10 a.m. The adrenal cortical activity was determined by a fluorimetric method measuring free 11-hydroxycorticoids in plasma (Mattingly, 1962). A normal series consisting of 10 patients had been studied previously and had given the following results: the mean value of the increase during the test was $16.0 \mu \mathrm{g} / 100 \mathrm{ml}$. and the geometric mean value 2 SD $9.0 \mu \mathrm{g} / 100 \mathrm{ml}$. An increase of less than $9.0 \mu \mathrm{g} / 100$ $\mathrm{ml}$. plasma is thus regarded as a pathological response to the test. The Metopirone test was performed according to the method described previously (Lundberg and Wide, 1969). An increase of less than $7 \cdot 7 \mathrm{mg} / 24$ hoursthat is, the geometric mean value for a control group -2SD_-is regarded as a pathological response to the test.

The plasma cortisol diurnal rhythm was studied by measurement of free 11-hydroxycorticoids in plasma every fourth hour for 24 hours.

\section{RESULTS}

CONTROL GROUP The mean value of the increase of free 11-hydroxycorticoids in plasma during the vasopressin test for the seven control patients was $12.7 \mu \mathrm{g} / 100 \mathrm{ml}$. In all control cases the value lay within \pm 2 SD of the mean found previously for normal persons.
GROUP I The results are given in the Table. In 11 of the patients in this optic atrophy group the result of the vasopressin test was pathological. In three of these (nos. 3, 5, and 7) duplicate tests were performed, and these gave the same results. In patient no. 8 the result of the vasopressin test was pathological after eight months of illness, while a further test 10 months later gave normal values. A pathological result was obtained for the patient with Leber's disease (no. 3) and for the three patients with tobacco-alcoholic amblyopia (nos. 4, 5, and 7). In three patients the test values were normal.

The Metopirone test was performed in 14 of the patients. The result of one patient's test was pathological, and in this case the vasopressin test also gave a pathological value. In the remaining patients the Metopirone tests gave normal values.

No clear relationships were found between the duration of the disease and the degree of visual loss, on the one hand, and the results of the tests, on the other. Pathological responses to vasopressin were found both in patients with bilateral and in those with unilateral optic atrophy.

GROUP II Of these patients with optic neuritis, three showed a pathological and two a normal result of the vasopressin test. The Metopirone test gave normal values in the two patients in whom this was performed (see Table).

\section{DISCUSSION}

The vasopressin test appears to be one of the most sensitive tests of disturbed hypothalamo-hypophyseal function. The applicability of the test has been documented in several reports. In a previous investigation conducted by the authors (Lundberg and Wide, 1969) with exactly the same method as in the present study, the test gave an abnormal result in eight of 19 patients with a hypophyseal tumour in situ, and in 19 of 22 patients in whom a hypophyseal tumour had been extirpated. When in the present investigation vasopressin tests were made on patients with optic atrophy not caused by tumour, a pathological response was obtained in 12 of 15 cases and further in three of the five patients with optic neuritis. The vasopressin test thus reveals a disturbed hypothalamo-hyphophyseal function but it cannot be used for differential diagnosis between optic disorders caused by tumour and those which are not.

The Metopirone test is also a useful aid in the diagnosis of hypothalamo-hypophyseal functional disturbances (for literature, see Lundberg and Hugosson, 1966). Out of 48 patients with tumours in the sellar region, the majority causing visual field 
TABLE

RESULTS OF TESTS IN GROUP I (15 PATIENTS WITH OPTIC ATROPHY) AND GROUP II (FIVE PATIENTS WITH OPTIC NEURITIS)

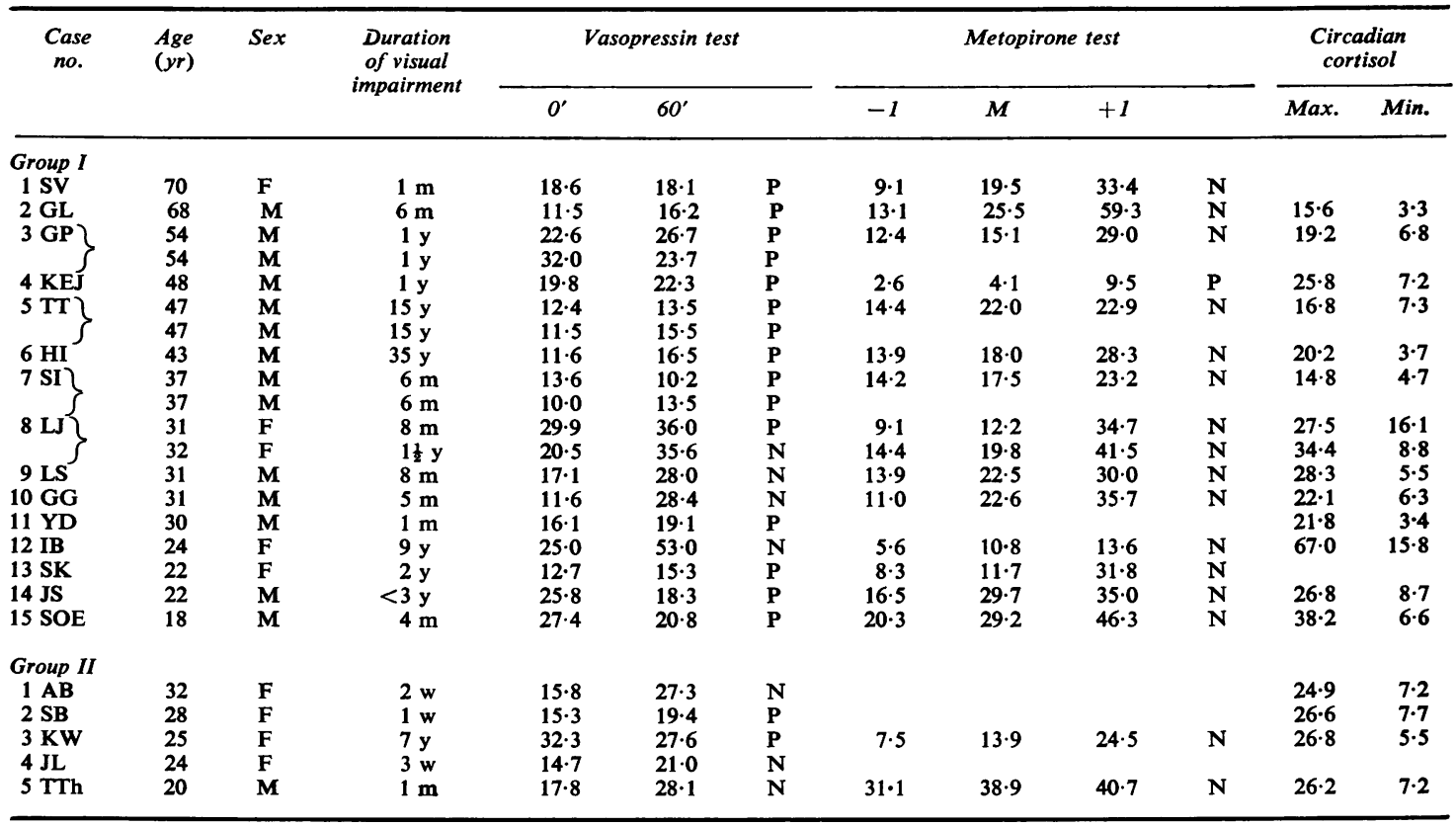

Vasopressin test: $0^{\prime}$ : just before infusion of lysine-8- vasopressin. $60^{\prime}: 60 \mathrm{~min}$ later, just after 'infusion of lysine-8- vasopressin. Assay of plasma free 11-hydroxycorticoids, expressed as $\mu \mathrm{g}$ cortisol/100 ml. plasma. P: pathological test. Metopirone test: M: day Metopirone was given. - 1: day before. +1: day after that. Assay of total 17-hydroxycorticosteroids in the urine, $\mathrm{mg} / 24 \mathrm{hr}$. P: pathological test. Circadian cortisol: Max: highest value of a $24 \mathrm{hr}$ curve. Min.: lowest value of a $24 \mathrm{hr}$ curve. Assay of plasma free 11-hydroxycorticoids, expressed as $\mu \mathrm{g}$ cortisol $/ 100 \mathrm{ml}$. plasma.

defects and visual impairment, the Metopirone test gave a pathological result in 23 cases and normal in 25 cases (Lundberg and Hugosson, 1966). In the present study, the Metopirone test was performed in 14 of the patients with optic atrophy and gave a pathological result in only one case. The higher frequency of pathological responses in the previous investigation of patients with tumours in the sellar region indicates that the Metopirone test might be of value in diagnosing tumours as a cause of optic atrophy. The difference in frequency of pathological responses between the vasopressin and the Metopirone tests in this study is also remarkable and may be due to differences in mode of action of the two tests.

The observation that among the patients with diseases in the optic nerve and chiasm so many had disturbances of the hypothalamo-hypophyseo-adrenal function may be of importance. It is true that in the majority of cases the functional reduction was not so severe that substitution needed to be considered, but nevertheless there would seem to have been some risk of adrenal cortical insufficiency in association with serious situations of stress, such as surgical operations.

It may be noted, further, that hypophyseal insufficiency, affecting the hypophyseo-adrenal function, has been described in a number of cases of traumatic brain damage (McCarthy, Wills, Keane, Gough, and Read, 1964; Rinne, 1966) and subarachnoid haemorrhage (Gerstenbrand and Weingarten, 1963; Jenkins, Buckell, Carter, and Westlake, 1969). The background to this insufficiency is considered to be a vascularly dependent, sometimes very minor necrosis or bleeding in the hypophysis (Daniel, Prichard, and Treip, 1959; Ceballos, 1966), or possibly in the hypothalamus.

The aetiology of the optic atrophy in the patients of our study was often obscure. Infarcts in the optic nerve have been described as a cause of optic atrophy (Ellenberger and Netsky, 1968). Only two of our 10 patients who underwent angiography had proven vascular lesions (here of the arteriosclerotic type). However, in seven of the patients the illness 
had an acute onset or occurred in attacks, which might indicate a vascular origin.

Investigations of the arterial vascular supply to the posterior part of the optic nerve, the chiasm, the basal anterior hypothalamus, and the hypophysis reveal considerable individual variations (McConell, 1953; Dawson, 1958). In most cases the anterior of the two superior hypophyseal arteries divides into one branch passing to the underside of the anterior part of the chiasm and the proximal part of the optic nerve, and another branch passing to the infundibulum and thereby to the superior capillary system of the portal vessels and to the anterior lobe of the hypophysis. This artery seems to be of importance for the entire hypothalamo-hypophyseal regulation. Theoretically, it is thus possible to obtain a vascular occlusion causing both an optic lesion and a disturbance of hypothalamo-hypophyseal function.

The pathological response to the vasopressin test in three of the five patients with retrobulbar neuritis based on disseminated sclerosis might be explained by a disturbed vascular supply or by a plaque in the hypothalamus.

\section{REFERENCES}

Bohm, E., and Dolk, Th. (1969). Kirurgisk behandling av hypofysadenom. Nord. Med., 82, 1237-1243.
Ceballos, R. (1966). Pituitary changes in head trauma. (Analysis of 102 consecutive cases of head injury.) Ala. J. med. Sci., 3, 185-198.

Daniel, P. M., Prichard, M. M. L., and Treip, C. S. (1959). Traumatic infarction of the anterior lobe of the pituitary gland. Lancet, 2, 927-931.

Dawson, B. H. (1958). The blood vessels of the human optic chiasma and their relation to those of the hypophysis and hypothalamus. Brain, 81, 207-217.

Ellenberger, C., Jr., and Netsky, M. G. (1968). Infarction in the optic nerve. J. Neurol. Neurosurg. Psychiat., 31, 606-611.

Gerstenbrand, F., and Weingarten, K. (1963). Aneurysma $\vec{F}$ und hypophysäres Syndrom. Wien. Z. Nervenheilk., 20, 300-310.

Jenkins, J. S., Buckell, M., Carter, A. B., and Westlake, S. (1969). Hypothalamic-pituitary-adrenal function after $\overline{\bar{S}}$ subarachnoid haemorrhage. Brit. med. J., 4, 707-709.

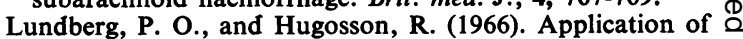
the Metopirone test to tumors in the region of the sella turcica. J. Neurosurg., 25, 543-548.

Lundberg, P. O., and Wide, L. (1969). Vasopressin test in $\vec{\circ}$ tumours of the sellar region. Neuroendocrinology, 5, 64-74.

McCarthy, C. F., Wills, M. R., Keane, P. M., Gough, K. R., $\vec{\omega}$ and Read, A. E. (1964). The Su-4885 (methopyrapone) response after head injury. $J$. clin. Endocr., 24, 121-124.

McConnell, E. M. (1953). The arterial blood supply of the human hypophysis cerebri. Anat. Rec., 115, 175-203.

Mattingly, D. (1962). A simple fluorimetric method for the estimation of free 11-hydroxycorticoids in human plasma J. clin. Path., 15, 374-379.

Rinne, U. K. (1966). Corticotrophin secretion in patien with head injuries examined by the Metopirone tes? $\rightarrow$ Psychiat. et Neurol. (Basel), 152, 145-155. 